\title{
Erratum to: Fault-Tolerant Quantum Secure Direct Communication Protocol Based On Decoherence-Free States
}

\author{
Yan-Bing Li • Ting-Ting Song • Wei Huang • \\ Wei-Wei Zhang
}

Published online: 14 March 2015

(C) Springer Science+Business Media New York 2015

\section{Erratum to: Int J Theor Phys (2015) 54:589-597 \\ DOI 10.1007/s10773-014-2251-1}

In the original manuscript, the last name of "Wei-Wei Zhang" was misspelled. Here we make the correction and apologize for the error.

The online version of the article can be found at http://dx.doi.org/10.1007/s10773-014-2251-1.

Y.-B. Li $(\varangle)$ · T.-T. Song · W. Huang · W.-W. Zhang

State Key Laboratory of Networking and Switching Technology, Beijing University of Posts and Telecommunications, Beijing 100876, China

e-mail: liyanbing1981@gmail.com

Y.-B. Li

Beijing Electronic Science and Technology Institute, Beijing 100070, China

Y.-B. Li

Department of Electrical Engineering and Computer Science, Northwestern University,

Evanston, IL 60208, USA 